\title{
CORRESPONDENCE
}

\section{Comment on: 'Paracentral acute middle maculopathy and acute macular neuroretinopathy following SARS-CoV-2 infection'}

(c) The Author(s), under exclusive licence to The Royal College of Ophthalmologists 2021, corrected publication 2021

Eye (2022) 36:1507-1509; https://doi.org/10.1038/s41433-021-01709-5

\section{TO THE EDITOR:}

We read with interest Virgo et al.'s article which reported one case each of paracentral acute middle maculopathy (PAMM) and typical acute macular neuroretinopathy (AMN) following Covid-19 infection [1]. We would like to report two symptomatic patients with bilateral findings of AMN following Covid-19 infection and one patient who developed unilateral AMN 3 weeks following Covid-19 vaccination (Pfizer-BioNTech vaccine). All these cases were serologically positive for Covid-19 antibody.

\section{CASE 1}

A 59-year-old male with type 2 diabetes mellitus presented with bilateral sudden onset of blurry vision, preceded by typical Covid-19 symptoms 2 weeks prior. On examination, his corrected visual acuity (CVA) was $6 / 9$ in both eyes. Ocular examination was unremarkable. Optical coherence tomography (OCT) revealed bilateral hyper-reflective bands in the outer plexiform layer (OPL) associated with loss of volume in the outer nuclear layer (ONL) and disruption to the ellipsoid zone, appearance suggestive of
AMN (Fig. 1a, b). At 6 weeks follow-up, the blurry vision had resolved subjectively but OCT appearance remained unchanged.

\section{CASE 2}

A 24-year-old, fit and well female patient reported 2-week history of paracentral scotomas in both eyes, preceded by typical Covid-19 symptoms 1 week before onset. CVA was 6/6 in both eyes. Examination revealed perifoveal dark gray patches. OCT changes were similar to Case 1 (Fig. 1c, d). A diagnosis of AMN was made. Unfortunately, her symptoms did not improve and OCT findings were unchanged 4 months later.

\section{CASE 3}

A 54-year-old male with well-controlled type 2 diabetes mellitus who was a low myope, presented with sudden onset of photopsias and a small scotoma in his left eye, 3 weeks after the first dose of Covid-19 vaccination (Pfizer-BioNTech). CVA was $6 / 6$ in both eyes. Fundal examination demonstrated an orange-brown oval-shaped lesion supero-temporal to the fovea with no other retinal pathology. OCT revealed a hyper-reflective band in the OPL and disruption of the interdigitation/ellipsoid zone, consistent with AMN (Fig. 1e). At 2 months follow-up,
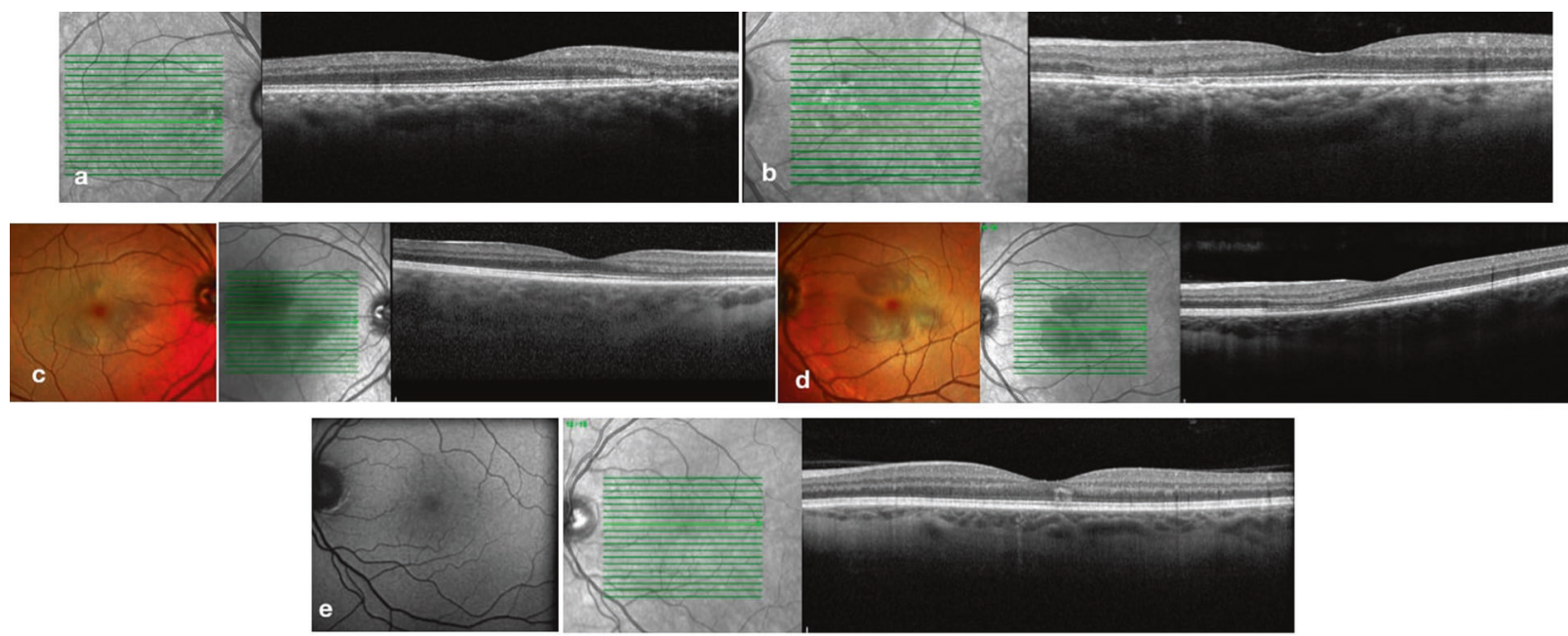

Fig. 1 Fundoscopic and Optical Coherence Tomography findings in patients with Covid-19-related AMN. a, b Case 1. Optical coherence tomography (OCT): bilateral hyper-reflective bands in the outer plexiform layer (OPL) associated with loss of volume in the outer nuclear layer $(\mathrm{ONL})$ and disruption to the ellipsoid zone in right eye (RE) and left eye (LE), respectively, $\mathbf{c}$, $\mathbf{d}$ Case 2 . Fundus photograph shows RPE changes. Infrared fundus photography reveals dark gray perifoveal lesions and OCT scans show thickening of the OPL and volume loss in the ONL nasal to the fovea associated with disruption of the interdigitation zone in RE and LE, respectively, e: Case 3. LE Fundus autofluorescence appears normal while OCT demonstrates hyper-reflective band at the level of OPL.

Received: 29 June 2021 Revised: 3 July 2021 Accepted: 16 July 2021

Published online: 2 August 2021 


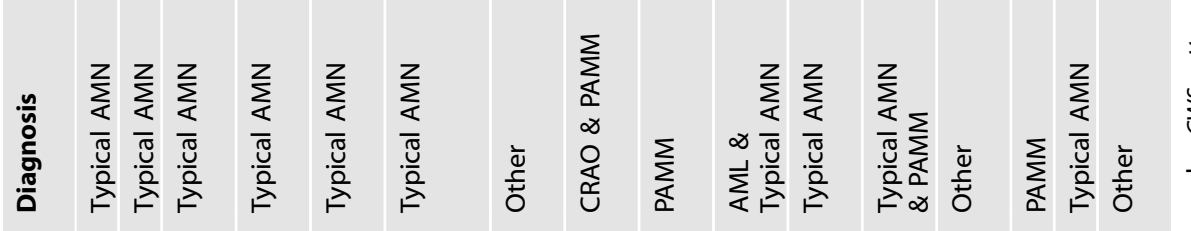

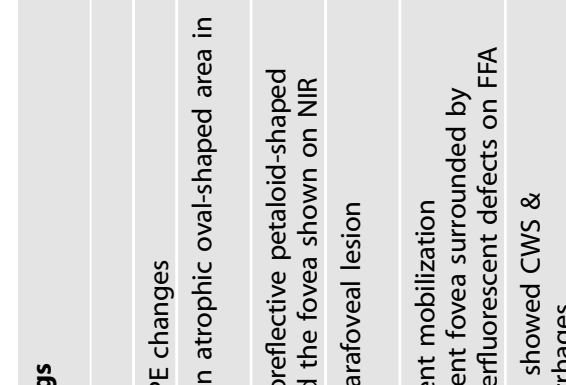

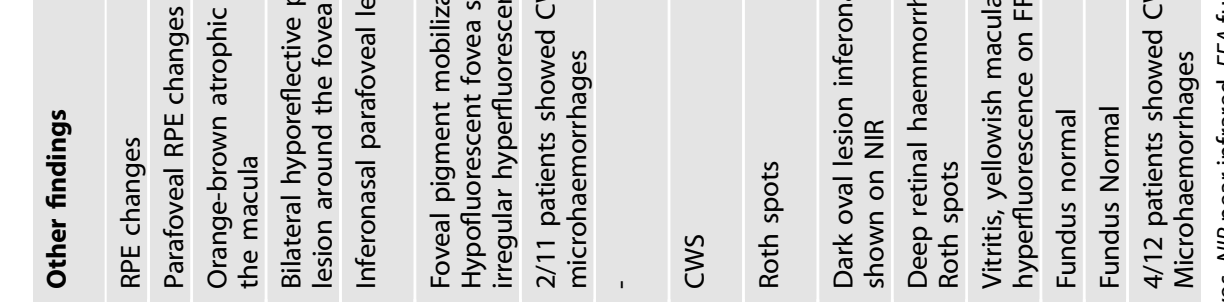

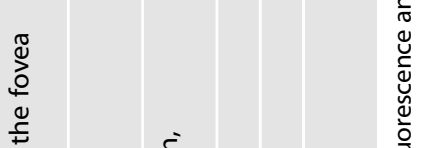

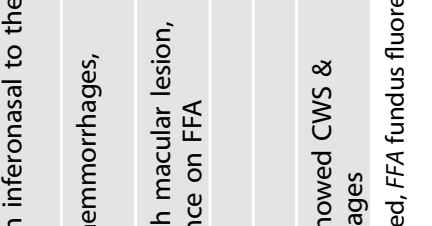

这

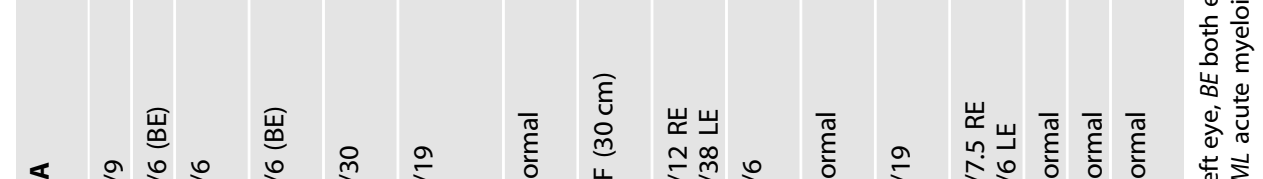
รา

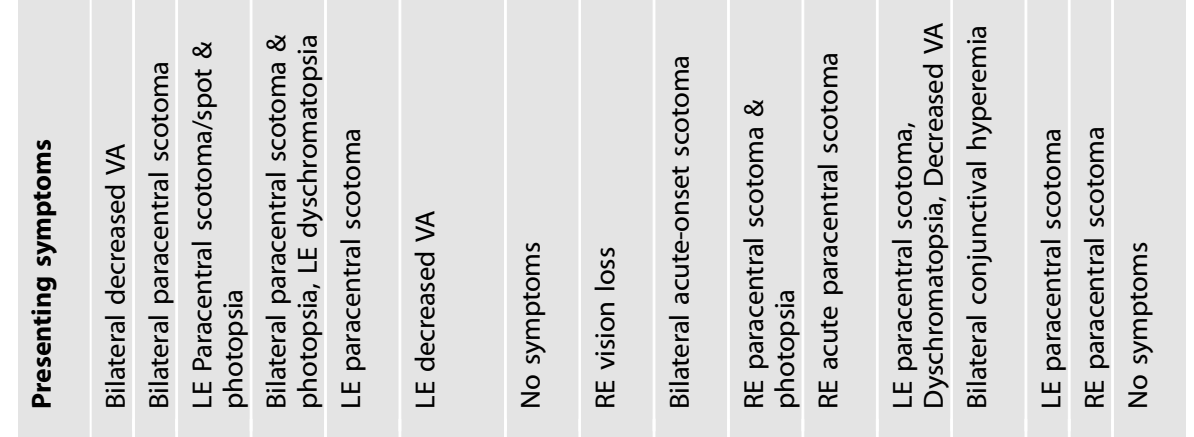

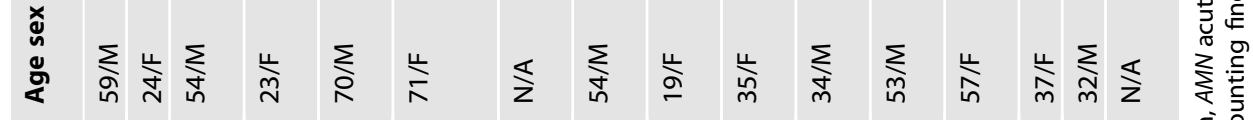

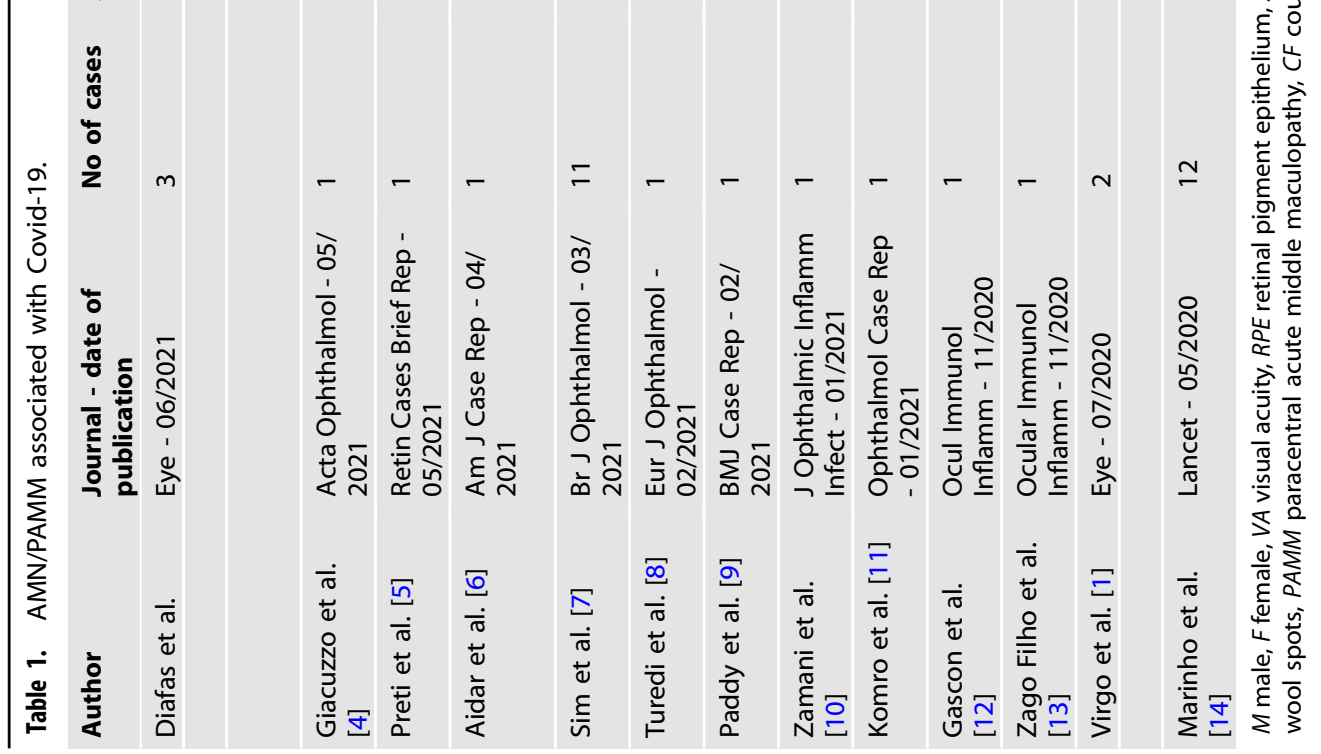


patient remained symptomatic but macular OCT showed complete resolution of the hyper-reflective band.

\section{DISCUSSION}

All of our patients presented with different severity of symptoms but retinal findings were consistent with the diagnosis of typical AMN [2], two following serologically proven Covid-19 infection, and one following Covid-19 vaccination. There have been several reports in the literature documenting AMN/PAMM in patients infected with Covid-19 (Table 1) but to the best of our knowledge there has been no other reports of this condition following Covid-19 vaccination. It did appear that the OCT changes in Case 3 following Covid-19 vaccination were less severe and demonstrated complete resolution, compared to Case 1 and Case 2. Further post-vaccination reports are needed before any relationship can be implicated to vaccination.

While the pathophysiology of AMN is not clear, a microvascular etiology seems to be implicated [2]. Although a true association between AMN/PAMM and Covid-19 infection remains unclear, microangiopathy and microvascular occlusion have been described in patients infected by Covid-19 [3]. Further studies are required to understand the link between AMN and COVID-19. Reporting vigilance should apply to cases of AMN/PAMM after Covid-19 vaccination.

Asterios Diafas $\mathbb{D}^{1 凶}{ }^{凶}$, Nima Ghadiri (iD ${ }^{1}$, Nick Beare (iD ${ }^{1,2}$ Savita Madhusudhan ${ }^{1,2}$, Ian Pearce ${ }^{1,2}$ and Shi Zhuan Tan ${ }^{1,2}$ ${ }^{1}$ St Paul's Eye Unit, Royal Liverpool University Hospital, Liverpool, UK. ${ }^{2}$ Department of Eye and Vision Science, University of Liverpool, Liverpool, UK. ${ }^{\circledR}$ email: asterisdiafas@gmail.com

\section{REFERENCES}

1. Virgo J, Mohamed M. Paracentral acute middle maculopathy and acute macular neuroretinopathy following SARS-CoV-2 infection. Eye. 2020;34:2352-3.

2. Bhavsar KV, Lin S, Rahimy E, Joseph A, Freund KB, Sarraf D, et al. Acute macular neuroretinopathy: a comprehensive review of the literature. Surv Ophthalmol. 2016;61:538-65.

3. Landecho MF, Yuste JR, Gándara E, Sunsundegui P, Quiroga J, Alcaide AB, et al. COVID-19 retinal microangiopathy as an in vivo biomarker of systemic vascular disease? J Intern Med. 2021;289:116-20.

4. Giacuzzo C, Eandi CM, Kawasaki A. Bilateral acute macular neuroretinopathy following COVID-19 infection. Acta Ophthalmol. 2021. https://doi.org/10.1111/ aos.14913.

5. Preti RC, Zacharias LC, Cunha LP, Monteiro MLR. Acute macular neuroretinopathy as the presenting manifestation of COVID-19 infection. Retin Cases Brief Rep. 2021. https://doi.org/10.1097//CB.0000000000001050.
6. Aidar MN, Gomes TM, de Almeida MZH, de Andrade EP, Serracarbassa PD. Low visual acuity due to acute macular neuroretinopathy associated with COVID-19: a case report. Am J Case Rep. 2021;30:22.

7. Sim R, Cheung G, Ting D, Wong E, Wong TY, Yeo I, et al. Retinal microvascular signs in COVID-19. Br J Ophthalmol. 2021. https://doi.org/10.1136/bjophthalmol2020-318236.

8. Turedi N, Onal Gunay B. Paracentral acute middle maculopathy in the setting of central retinal artery occlusion following COVID-19 diagnosis. Eur J Ophthalmol. 2021. https://doi.org/10.1177/1120672121995347.

9. Padhy SK, Dcruz RP, Kelgaonkar A. Paracentral acute middle maculopathy following SARS-CoV-2 infection: the D-dimer hypothesis. BMJ Case Rep. 2021;14: e242043.

10. Zamani G, Ataei Azimi S, Aminizadeh A, Shams Abadi E, Kamandi M, Mortazi H, et al. Acute macular neuroretinopathy in a patient with acute myeloid leukemia and deceased by COVID-19: a case report. J Ophthalmic Inflamm Infect. 2021;10:39.

11. Komro J, Bogaard JD, Warren CC. Acute macular neuroretinopathy as a manifestation of Covid-19. Ophthalmol Case Rep. 2021;5:1-1.

12. Gascon P, Briantais A, Bertrand E, Ramtohul P, Comet A, Beylerian M, et al. Covid-19associated retinopathy: a case report. Ocul Immunol Inflamm. 2020;28:1293-7.

13. Zago Filho LA, Lima LH, Melo GB, Zett C, Farah ME. Vitritis and outer retinal abnormalities in a patient with COVID-19. Ocul Immunol Inflamm. 2020;28: 1298-300.

14. Marinho PM, Marcos AAA, Romano AC, Nascimento $H$, Belfort $R$ Jr. Retinal findings in patients with COVID-19. Lancet. 2020;395:1610.

\section{AUTHOR CONTRIBUTIONS}

All authors contributed to the study conception and design. Patient's examination, diagnosis and treatment were performed by Dr Nima Ghadiri, Prof lan Pearce and Dr Shi Zhuan Tan. The original draft of the manuscript was written by Dr Asterios Diafas. Dr Diafas also conducted a thorough literature search. Dr Nicholas Beare contributed to data collection and presentation. Dr Shi Zhuan Tan and Dr Savita Madhusudhan supervised research activity planning and execution. All authors read, commented, and approved the final version of the manuscript.

\section{COMPETING INTERESTS}

The authors declare no competing interests.

\section{ADDITIONAL INFORMATION}

Correspondence and requests for materials should be addressed to A.D.

Reprints and permission information is available at http://www.nature.com/ reprints

Publisher's note Springer Nature remains neutral with regard to jurisdictional claims in published maps and institutional affiliations. 